\title{
Análisis del efecto de la crisis en la contratación laboral por grupos de ocupación en Andalucía*
}

\author{
M. ALEJANDRO CARDENETE FLORES \\ Comisión Europea (Joint Research Centre - Institute for Prospective Technological Studies). \\ Departamento de Economía,UNIVERSIDAD PABLO DE OLAVIDE, ESPAÑA. E-mail: \\ macardenete@upo.es
}

\section{PATRICIA D. FUENTES SAGUAR}

Departamento de Economía, UNIVERSIDAD PABLO DE OLAVIDE, ESPAÑA. E-mail: pfuesag@upo.es

\author{
ALFREDO J. MAINAR CAUSAPÉ \\ Departamento de Economía Aplicada III, UNIVERSIDAD DE SEVILLA, ESPAÑA. E-mail: \\ amainar@us.es
}

\section{RESUMEN}

El objetivo de este trabajo es analizar los efectos de la actual crisis económica en la estructura del mercado de trabajo de Andalucía, utilizando como herramienta un modelo multisectorial. Para ello se realiza un estudio de dicho mercado de trabajo en cuanto a ramas de actividad y grupos de ocupación, determinando cuales son los que presentan un mayor número de contratos, tanto agregada, como sectorialmente, en Andalucía, ante crecimientos o decrecimientos económicos sectoriales o generales. Se toman aquí los contratos celebrados como variable aproximada de la creación de empleo, realizando un análisis de la relación entre impactos exógenos en sectores de actividad y generación de contratos laborales, así como de su distribución entre los principales grupos de ocupación. La principal herramienta de análisis para lograr este objetivo será el uso de una Matriz de Contabilidad Social para Andalucía (año 2005), aplicando el análisis lineal de multiplicadores y extendiéndolo al estudio de ocupaciones y contratos.

Palabras clave: Mercado de trabajo, Matriz de Contabilidad Social, Modelos SAM, Análisis de impactos.

\section{Impact Analysis of the Economic Crisis in the Recruitment by Occupational Groups in Andalusia}

\begin{abstract}
The aim of this paper is to analyze the effects of the current economic crisis on the labor market structure of Andalusia, using a multisectoral model. This is a study of the labor market in terms of industry and occupational groups, determining their evolution, both aggregate and sectoral, in Andalusia, due to economic growth or recession. It presents the contracts as a proxy for job creation, analyzing the relationship between exogenous shocks in sectors of activity and generation of employment contracts, and their distribution among the major occupational groups. The main analysis tool for achieving this goal is the use of a Social Accounting Matrix for Andalusia (2005), applying linear multiplier analysis and extending the study of occupations and contracts.
\end{abstract}

Keywords: Labour market, Social Accounting Matrix, SAM Linear Models, Impact Analysis.

Clasificación JEL: D57, J20

* Los autores son los únicos responsables del presente texto, sin ninguna implicación de la Comisión Europea.

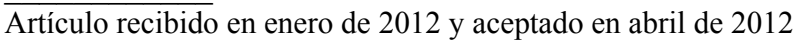

Artículo disponible en versión electrónica en la página www.revista-eea.net, ref. ə-30119 


\section{INTRODUCCIÓN Y OBJETIVOS}

El mercado de trabajo en España está caracterizado por sus elevados ritmos de creación y destrucción de empleo. La aguda situación de crisis en la que está inmersa nuestra economía en este momento está causando una rápida destrucción de puestos de trabajo. Ante esta situación, resulta especialmente importante conocer de forma más precisa cómo se ven afectadas las diferentes ramas económicas, sectores o grupos de ocupación, intentando cuantificar las relaciones entre actividad sectorial y empleo. Esto permitirá conocer mejor en qué sectores se debe actuar si se quiere potenciar determinados tipos de empleo dirigidos a colectivos específicos. Por ello, en este trabajo se busca determinar qué actividades y grupos de ocupación son los que presentan un mayor número de contratos (como variable aproximada de la creación de empleo), tanto agregada, como sectorialmente, en Andalucía, y cómo responden cuantitativamente ante impactos en diferentes sectores. Para ello, se utiliza una descripción inicial, de la distribución de estos contratos y su estructura al comienzo de la crisis, realizando a continuación un análisis de la relación entre impactos exógenos en sectores de actividad y generación de contratos laborales, así como de su distribución entre los principales grupos de ocupación.

Para este análisis se recurre al uso de un modelo multisectorial de tipo lineal, basado en la Matriz de Contabilidad Social de Andalucía del año 2005 (Cardenete et al. 2010, Cardenete y Fuentes, 2009). Este modelo que aquí se presenta, que amplía las posibilidades de otros planteamientos de tipo input-output, supone la creación de una herramienta de análisis y simulación de gran utilidad para la evaluación de posibles escenarios y su impacto sobre la economía y el empleo. Teniendo en cuenta que la creación de empleo es una de las prioridades de cualquier política económica actual y que es creciente la necesidad de incidir en actuaciones concretas, tanto generales, como sectoriales, por parte de los gobiernos nacional y autonómico, este tipo de instrumento analítico resulta de especial interés. Si, además, se tiene en cuenta que las nuevas políticas están sometidas a las estrictas restricciones presupuestarias a las que conlleva la prioridad de control del déficit, el conocimiento de los efectos de los impactos sectoriales de dichas acciones, utilizando ejercicios como el aquí presentado, resulta esencial para decidir hacia dónde deben dirigirse los escasos recursos.

\section{ESTRUCTURA Y EVOLUCIÓN DE LA CONTRATACIÓN EN ANDALUCÍA EN EL INICIO DE LA CRISIS}

Existen diversos trabajos que analizan los datos de empleo y el mercado laboral en Andalucía, si bien los análisis sobre datos más recientes pueden encontrarse en el Observatorio ARGOS de la Junta de Andalucía o en trabajos como el de Ariza et al. (2011). Sin embargo, en este trabajo se pretende centrar el estudio en los comienzos de la crisis económica actual, aproximando su im- 
pacto a través de la evolución de las contrataciones. De esta forma, para comprender mejor el efecto de la crisis en la contratación laboral en Andalucía, un primer paso obligado es describir la situación al inicio de la misma, centrando el análisis en las ramas productivas y en los distintos tipos de ocupación, así como en las relaciones entre ambos.

Con el fin de poder analizar posteriormente los efectos o impactos que posibles aumentos en la actividad de las ramas productivas pueden tener en los diferentes grupos de ocupación considerados, se ha obtenido (Tabla 1) la distribución de los contratos por dichos grupos para distintas ramas o secciones de actividad (en función de la disponibilidad de datos y de su operatividad).

Tabla 1

Distribución de los contratos laborales realizados por grupos de ocupación, según sectores de actividad. Andalucía. Años 2007 y 2008

\begin{tabular}{|c|c|c|c|c|c|c|c|c|c|c|}
\hline & S1 & S2 & S3 & S4 & S5 & s6 & S7 & S8 & s9 & $\begin{array}{c}\text { Distribución } \\
\text { total por } \\
\text { ocupaciones }\end{array}$ \\
\hline G1 & $0,01 \%$ & $0,47 \%$ & $0,50 \%$ & $0,30 \%$ & $0,07 \%$ & $0,32 \%$ & $0,76 \%$ & $0,30 \%$ & $0,32 \%$ & $0,20 \%$ \\
\hline G2 & $0,05 \%$ & $3,38 \%$ & $4,10 \%$ & $1,64 \%$ & $0,94 \%$ & $1,53 \%$ & $0,75 \%$ & $3,31 \%$ & $20,60 \%$ & $3,37 \%$ \\
\hline G3 & $0,26 \%$ & $6,66 \%$ & $5,63 \%$ & $5,34 \%$ & $1,55 \%$ & $7,01 \%$ & $3,31 \%$ & $8,65 \%$ & $14,71 \%$ & $5,20 \%$ \\
\hline G4 & $0,13 \%$ & $6,62 \%$ & $10,76 \%$ & $5,35 \%$ & $1,95 \%$ & $12,29 \%$ & $27,78 \%$ & $12,19 \%$ & $5,50 \%$ & $6,58 \%$ \\
\hline G5 & $0,12 \%$ & $1,25 \%$ & $3,26 \%$ & $4,00 \%$ & $0,22 \%$ & $43,31 \%$ & $4,95 \%$ & $33,75 \%$ & $21,83 \%$ & $15,83 \%$ \\
\hline G6 & $30,17 \%$ & $3,33 \%$ & $1,05 \%$ & $0,73 \%$ & $1,49 \%$ & $2,59 \%$ & $0,17 \%$ & $0,48 \%$ & $4,41 \%$ & $9,51 \%$ \\
\hline G7 & $0,18 \%$ & $20,33 \%$ & $15,41 \%$ & $41,47 \%$ & $64,66 \%$ & $7,61 \%$ & $1,56 \%$ & $2,07 \%$ & $6,31 \%$ & $15,07 \%$ \\
\hline G8 & $1,64 \%$ & $41,06 \%$ & $14,53 \%$ & $16,41 \%$ & $3,42 \%$ & $7,28 \%$ & $47,97 \%$ & $3,08 \%$ & $1,38 \%$ & $4,95 \%$ \\
\hline G9 & $67,43 \%$ & $16,89 \%$ & $44,76 \%$ & $24,75 \%$ & $25,69 \%$ & $18,07 \%$ & $12,75 \%$ & $36,18 \%$ & $24,94 \%$ & $39,29 \%$ \\
\hline $\begin{array}{c}\text { Distribución } \\
\text { total por sectores }\end{array}$ & $27,95 \%$ & $0,08 \%$ & $0,14 \%$ & $4,28 \%$ & $17,52 \%$ & $8,05 \%$ & $3,18 \%$ & $29,08 \%$ & $9,72 \%$ & $100 \%$ \\
\hline
\end{tabular}

G1 Dirección de las empresas y de las administraciones públicas

G2 Técnicos y profesionales científicos e intelectuales

G3 Técnicos y profesionales de apoyo

G4 Empleados de tipo administrativo

G5 Trabajadores de los servicios de restauración, personales, protección y vendedores de los comercios

G6 Trabajadores cualificados en la agricultura y en la pesca

G7 Artesanos y trabajadores cualificados de las industrias manufactureras, la construcción, y la minería,excepto los operadores de instalaciones y maquinaria

G8 Operadores de instalaciones y maquinaria y montadores

G9 Trabajadores no cualificados

S1 Agricultura y pesca

S2 Industrias extractivas

S3 Producción y distribución de energía eléctrica, gas y agua

S4 Industria manufacturera

S5 Construcción

Fuente: Elaboración propia a partir de datos del Ministerio de Empleo y Seguridad Social.
S6 Comercio

S7 Transporte y comunicaciones

S8 Otros servicios destinados a la venta

S9 Servicios no destinados a la venta

Esta distribución muestra una clara preponderancia de los contratos a Trabajadores no cualificados en Otros servicios destinados a la venta (36\% del total), Producción y distribución de energía eléctrica, gas y agua (45\%) y, 
especialmente, Agricultura y pesca, sector en el que suponen más del $67 \%$ del total de contratos. También son importantes estos contratos, con porcentajes alrededor del $25 \%$ del total de los realizados, en los sectores de Industria Manufacturera, Construcción y Servicios no destinados a la venta. No obstante, hay que tener en cuenta que el empleo de trabajadores con esta cualificación suele estar caracterizado por la sucesión de contratos temporales realizados directamente por el empresario o mediante el uso de empresas de trabajo temporal, especialmente en algunos sectores, como es el caso de Otros servicios destinados a la venta y Agricultura, lo que aumenta el peso de dichas ocupaciones sobre el total.

Los Operadores de instalaciones,... acaparan el $41 \%$ y el $48 \%$, respectivamente, de los contratos en los sectores Industrias Extractivas y Transporte y comunicaciones, mientras que Artesanos y trabajadores cualificados... es el grupo de ocupación mayoritaria en la Industria manufacturera (41\%) y, de forma abrumadora, en Construcción (65\%), pudiendo estar igualmente explicado por la sucesión de contratos temporales en este sector. Los contratos a Trabajadores de los servicios,... son mayoritarios en Comercio (43\%) y en Otros servicios destinados a la venta (34\%), teniendo también un porcentaje importante (22\%) en Servicios no destinados a la venta. Sin embargo, este último sector destaca especialmente por la elevada proporción (21\%) de contratos a Técnicos y profesionales científicos e intelectuales, lo que viene explicado por la inclusión en esta rama de actividad de los servicios públicos de sanidad y educación.

\section{Evolución de la contratación entre 2007 y 2009}

En la sección anterior se ha analizado la estructura de la contratación en Andalucía al comienzo de la crisis, en lo que a ramas de actividad y grupos de ocupación se refiere. A continuación se realiza un breve repaso de su evolución en estos años, relacionando la contratación en Andalucía con las variaciones en el PIB a precios de mercado de la economía regional. Se amplía el ámbito temporal de estudio hasta 2009 para recoger la fuerte recesión sufrida en ese periodo.

Así, en el Gráfico 1 se resumen conjuntamente las evoluciones respecto a 2006 del PIB pm andaluz y del total de contrataciones realizadas en la comunidad autónoma. En él se comprueba que la producción interior cae en 2009 hasta niveles de 2006, fruto del estancamiento de 2008 (incremento del PIB del $0,6 \%$ ) y de la fuerte recesión de 2009 (caída del -3,6\%), que se ve reflejada en un fortísimo descenso en el número de contrataciones, inferiores en casi un $16 \%$ a las del año 2006. 


\section{Gráfico 1}

Evolución del Producto Interior Bruto a precios de mercado (PIB pm) y del total de contratos $(2006=100)$. Andalucía

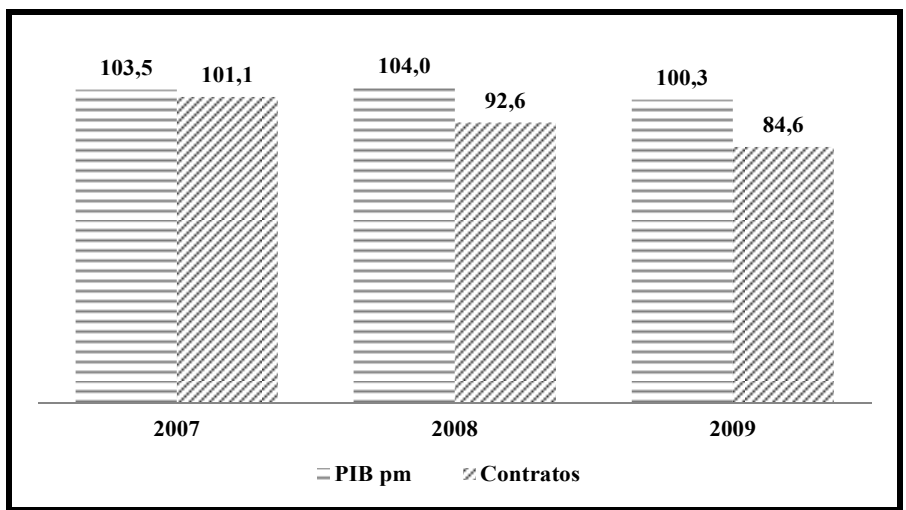

Fuente: Sistema de Información de los Servicios Públicos de Empleo (SISPE). Instituto de Estadística y Cartografía de Andalucía (IECA). Elaboración propia.

\section{Tabla 2}

Evolución anual del Valor Añadido Bruto (VAB) sectorial y del PIB pm regional en Andalucía

\begin{tabular}{llll}
\cline { 2 - 4 } & $\mathbf{2 0 0 7}$ & $\mathbf{2 0 0 8}$ & $\mathbf{2 0 0 9}$ \\
\hline VAB Ramas primarias & $2,6 \%$ & $0,0 \%$ & $2,1 \%$ \\
VAB Ramas industriales & $-0,4 \%$ & $0,2 \%$ & $-9,2 \%$ \\
VAB Construcción & $2,2 \%$ & $-2,6 \%$ & $-13,2 \%$ \\
Servicios & $4,8 \%$ & $1,6 \%$ & $-0,8 \%$ \\
\hline PIB pm & $\mathbf{3 , 5 \%}$ & $\mathbf{0 , 6} \%$ & $\mathbf{- 3 , 6 \%}$ \\
\hline
\end{tabular}

Fuente: Instituto de Estadística y Cartografía de Andalucía (IECA).

La Tabla 3 muestra la evolución de las contrataciones de forma más deta1lada. En ella se observa que el sector Construcción (-2,6\% y $-13,2 \%$ en el VAB de 2008 y 2009, respectivamente, ver Tabla 2) no alcanzó en 2009 ni el 60\% de las contrataciones realizadas en el año 2006. Pero si el impacto de la crisis en este sector ha sido terrible, las ramas industriales, que ya venían presentando contracción desde el año 2006, se desploman en 2009, con una caída en el VAB del $-9,2 \%$. Los efectos en las contrataciones de las industrias manufactureras y energéticas se muestran en unos niveles apenas superiores al $83 \%$ respecto a 2006 , si bien este descenso proviene ya del año 2008 (85,9\% y 86,8\%, respectivamente). Además, el efecto conjunto del decrecimiento en construcción e industria conlleva una importante caída de la demanda de inputs de otros sec- 
tores, lo que provoca un descenso dramático en el número de contrataciones que realizan, como es el caso de Industrias Extractivas, que en 2009 efectuó poco más de la mitad de las contrataciones que hizo en 2006.

\section{Tabla 3}

Evolución de la contratación en Andalucía (2006=100).

Ramas de actividad y grupos de ocupación

\begin{tabular}{|c|c|c|c|}
\hline & \\
\hline & 2007 & 2008 & 2009 \\
\hline Agricultura y pesca & 104,0 & 103,4 & 100,8 \\
\hline Industrias extractivas & 84,6 & 73,7 & 53,9 \\
\hline Producción y distribución de energía eléctrica, gas y agua & 99,6 & 86,8 & 83,2 \\
\hline Industria manufacturera & 100,3 & 85,9 & 83,2 \\
\hline Construcción & 95,1 & 74,2 & 59,7 \\
\hline Comercio & 96,3 & 82,3 & 68,5 \\
\hline Transporte y comunicaciones & 98,7 & 88,8 & 91,4 \\
\hline Otros servicios destinados a la venta & 102,5 & 96,0 & 83,0 \\
\hline Servicios no destinados a la venta & 107,0 & 106,0 & 110,9 \\
\hline Dirección de las empresas y de las administraciones públicas & 102,2 & 90,8 & 82,4 \\
\hline Técnicos y profesionales científicos e intelectuales & 105,1 & 103,0 & 93,1 \\
\hline Técnicos y profesionales de apoyo & 114,6 & 111,7 & 100,9 \\
\hline Empleados de tipo administrativo & 102,6 & 91,9 & 71,5 \\
\hline $\begin{array}{l}\text { Trabajadores de los servicios de restauración, personales, protección y } \\
\text { vendedores de los comercios }\end{array}$ & 105,7 & 101,2 & 94,4 \\
\hline Trabajadores cualificados en la agricultura y en la pesca & 97,3 & 85,5 & 75,4 \\
\hline $\begin{array}{l}\text { Artesanos y trabajadores cualificados de las industrias manufactureras, la } \\
\text { construcción,... }\end{array}$ & 96,1 & 77,1 & 61,9 \\
\hline Operadores de instalaciones y maquinaria y montadores & 99,3 & 87,9 & 79,1 \\
\hline Trabajadores no cualificados & 100,7 & 95,9 & 93,5 \\
\hline Total & 101,1 & 92,6 & 84,6 \\
\hline
\end{tabular}

Fuente: Sistema de Información de los Servicios Públicos de Empleo (SISPE). Elaboración propia.

Finalmente, la recesión que también experimentan los servicios en 2009 ($0,8 \%$ ), lleva también a un significativo descenso en los contratos realizados en el sector Comercio, que en dicho año caen hasta el $68,5 \%$ de los efectuados en 2006, resintiéndose también del descenso general de la economía, como ocurre con el sector de Otros servicios destinados a la venta (con una caída del 17\% de las contrataciones entre 2006 y 2009). Las excepciones vienen dadas por Agricultura y pesca, que mantiene el nivel de contratación, y Servicios no destinados a la venta, que incluso los aumenta en un $10 \%$, como consecuencia de los esfuerzos del sector público por actuar de forma directa sobre los niveles de empleo.

En cuanto a la evolución de la contratación por grupos de ocupación, la crisis del sector de la Construcción tiene su reflejo en el grupo de ocupación Arte- 
sanos y trabajadores cualificados... (en el que se incluyen los trabajadores con alguna especialización), que experimenta un descenso muy similar al del sector. Por otra parte, cabe destacar que, el grupo de Trabajadores sin cualificación, significativo también en la Construcción, aguanta mejor la situación, apenas cayendo un $6,5 \%$.

También destaca significativamente el descenso de la contratación de Empleados de tipo administrativo y de Trabajadores cualificados en Agricultura y pesca, con descensos entre el $25 \%$ y el $30 \%$ respecto a 2006 en ambos casos. Aunque el dato para el primero de estos grupos de ocupación viene explicado por el descenso generalizado en la actividad de todos los sectores, incluidos los servicios en 2008, en el caso de los segundos contrasta con el mantenimiento de la contratación en el sector primario, si bien esto puede explicarse con el mantenimiento de la cifra de contratos a Trabajadores no cualificados, que conforman, como mostraba la Tabla 1, la mayor parte de los realizados en las ramas de Agricultura y pesca.

Por otra parte, la caída en la industria también implica una reducción de casi un $21 \%$ en los contratos celebrados con Operadores de instalaciones y maquinaria y montadores. Sin embargo, destacan especialmente el mantenimiento de los contratos en el grupo Técnicos y profesionales de apoyo, y la caída, más suave que en el resto, en Técnicos y profesionales cientificos e intelectuales, basados en el aumento de la contratación en el sector de Servicios no destinados a la venta.

\section{ANÁLISIS DEL IMPACTO DE LA ACTIVIDAD ECONÓMICA EN LA CONTRATACIÓN MEDIANTE UN MODELO MULTISECTORIAL DE LA ECONOMÍA ANDALUZA}

\section{Metodología de análisis. Ampliación del modelo lineal basado en la Matriz de Contabilidad Social}

Para analizar la forma en que las variaciones en la actividad de una determinada rama productiva generan contratación en uno u otro grupo de ocupación, se ha combinado la información procedente de las estadísticas de contratación existentes (facilitadas por CC.OO. Andalucía y obtenidas del Ministerio de Empleo y Seguridad Social) con la Matriz de Contabilidad Social de Andalucía para el año 2005, estimada en trabajos de investigación previos (Cardenete et al., 2010, Cardenete y Fuentes, 2009).

Una Matriz de Contabilidad Social (MCS) es una base de datos que recoge y organiza en una matriz cuadrada la información económica y social de todas las transacciones entre todos los agentes de una economía en un momento determinado del tiempo. El origen de las Matrices de Contabilidad Social está en el 
intento de integrar las estadísticas sociales en el Modelo Input-Output de interdependencia de los sectores productivos, representando de forma matricial una ampliación de estos modelos. Las tablas input output recogen la interdependencia de los sectores productivos y su relación con la demanda final, pero las Matrices de Contabilidad Social incorporan además todas las transacciones que se realizan entre los factores productivos y los componentes de la demanda final, ampliando con ello la información que ofrecen las tablas input-output y completando el flujo circular de la renta en una matriz cuadrada. Esta es la razón por las que en este trabajo se utiliza una MCS en lugar de hacer uso directamente de las tablas input output (opción perfectamente viable e igualmente válida).

En cuanto a los análisis a partir de esta herramienta, los inicios están en Stone (1962), y Pyatt y Round (1979), entre otros, iniciándose las aplicaciones en España a partir de trabajos como los de Kehoe et al. (1988) o Polo et al. (1991). Antecedentes de su aplicación a la economía andaluza pueden verse en Cardenete et al. (2008) y en Cardenete y Fuentes (2009), mientras que análisis de este tipo referidos a impactos económicos pueden encontrarse en Cámara et al. (2011) o en Polo y Valle (2011), entre otros.

A partir de la Matriz de Contabilidad Social de Andalucía se ha calculado la correspondiente matriz de multiplicadores contables, $\mathbf{M}$, que permite evaluar el impacto final (en euros) que, tras circular por el sistema económico, generan impactos exógenos (shocks) unitarios en la demanda de estos sectores.

La formulación de los modelos lineales de los que se obtienen los multiplicadores parte de la expresión general siguiente:

$$
\mathbf{y}_{\mathrm{n}}=\mathbf{A}_{\mathrm{n}} \mathbf{y}_{\mathrm{n}}+\mathbf{x}=\left(\mathbf{I}-\mathbf{A}_{\mathrm{n}}\right)^{-1} \mathbf{x}=\mathbf{M} \mathbf{x}
$$

donde $\mathbf{y}_{\mathrm{n}}$ es el vector columna de rentas de las cuentas endógenas, $\mathbf{A}_{\mathrm{n}}$ es la matriz de propensiones medias al gasto de las diferentes cuentas endógenas, cuyos componentes $a_{i j}$ representan el gasto realizado en la cuenta $i$ por cada unidad monetaria de gasto o empleo total de $j ; \mathbf{x}$ es el vector que contabiliza el total de los flujos de renta que las cuentas endógenas reciben de las cuentas exógenas.

La matriz $\mathbf{M}$ es la matriz de multiplicadores contables, cuyos componentes $\left(m_{i j}\right)$ reflejan el impacto que una unidad exógena de renta sobre una cuenta endógena $j$, genera finalmente sobre la renta de la cuenta endógena $i$. La elección de las variables endógenas depende de los objetivos del análisis y aquí se consideran como tal sólo a las actividades productivas, quedando el resto (los factores de producción, los sectores institucionales de consumo privado, Ahorro/Inversión, Gobierno y Sector Exterior) como cuentas exógenas.

Si a esta información se incorpora la procedente de las bases de datos de contratación, presentada en el apartado anterior, que permite descomponer los contratos de cada rama de actividad según los grupos de ocupación del puesto 
de trabajo relacionado, puede determinarse cómo un shock en uno de los sectores considerados puede generar contratos de trabajo de uno u otro grupo de ocupación. Así, si $\mathbf{H}$ es la matriz con tantas filas como grupos de ocupación considerados y con tantas columnas como ramas de actividad analizadas, distribuyendo porcentualmente para las segundas los contratos realizados según cada categoría de las primeras y c es el vector columna de contratos totales realizados por cada unidad monetaria (en este caso millones de euros) de empleos o recursos de un sector de actividad (siendo $\mathbf{C}$ su versión de matriz diagonal), puede obtenerse la matriz $\mathbf{Z}$.

$$
\mathbf{Z}=\mathbf{H ~ C ~ M ~}
$$

Cada elemento de esta matriz $\mathbf{Z}$ recoge el número de contratos generado para el grupo de ocupación $g$ (fila) por un impacto unitario (millón de euros) en el sector de actividad $i$ (columna).

\section{Efectos de impactos sectoriales en la contratación. Diferencias entre gru- pos de ocupación}

La aplicación de la metodología propuesta ${ }^{1}$ se ilustra con los datos resumidos en la Tabla 4. En dicha tabla se pueden observar los contratos que generaría en cada sector un shock exógeno de renta de un millón de euros en Andalucía. Hay que tener en cuenta que esta cuantificación puede aplicarse también, aunque en sentido negativo, en el caso de un descenso en la actividad del sector. De esta forma pueden comprenderse mejor los resultados obtenidos en la simulación principal presentada en el trabajo.

El sector agrícola, por sus especiales características en los sistemas de contratación y cotización, es el que más contratos generaría, 95 . Le siguen el sector de la Construcción (29 contratos) y Servicios, tanto los públicos, como los de carácter privado (especialmente los segundos), en los que se generarían 23 y 30 contratos, respectivamente. Si el shock exógeno fuera simultáneo en todos los sectores, el total de contratos generado sería de 228 con una buena parte de la contratación generada (47\%) destinada a Trabajadores sin cualificación. Estos resultados pueden interpretarse en sentido contrario, como descenso en la contratación, por lo que este colectivo sería el más afectado por un descenso de la actividad económica de los sectores, como ha ido sucediendo en los últimos años.

Al considerar los datos desagregados por sectores, el uso de la metodología presentada permite observar el efecto final (y no el meramente directo) de los

\footnotetext{
${ }^{1}$ Los datos de c y M corresponden al año 2005, mientras que $\mathrm{H}$ se ha calculado con los datos detallados de los años 2007 y 2008 , no afectando esto a la validez de los resultados puesto que dicha matriz recoge una estructura de demanda de trabajo (en términos de tipo de ocupación por sector) perfectamente aplicable en el corto plazo.
} 
impactos sectoriales exógenos en los tipos de ocupación contratados. Así, se observa que la consideración del conjunto del ciclo económico supone cambios en la composición relativa de la contratación generada por cada sector. En Andalucía, destaca el caso de las Industrias extractivas, en las que la contratación mayoritaria finalmente generada se corresponde con Trabajadores no cualificados, en detrimento (siempre en términos relativos) de los Artesanos y técnicos cualificados... y de Operadores de instalaciones y maquinarias, cuya contratación era la más significativa al considerar la directamente producida en el sector. Algo similar ocurre con las Industrias manufactureras. Resulta significativo el grupo de ocupación Trabajadores de los servicios de restauración, personales, protección y vendedores de los comercios, que se ve favorecido en cuanto a su participación en la contratación generada en sectores como Industrias extractivas o Producción y distribución de energía eléctrica, gas y agua, mientras que desciende la relevancia en el que podría considerarse su sector natural, Comercio. También es destacable la importancia relativa que adquieren los contratos a Trabajadores sin cualificación.

Tabla 4

Impacto (contratos generados) de un shock unitario (millón de euros) en cada sector. Andalucía

\begin{tabular}{|c|c|c|c|c|c|c|c|c|c|c|}
\hline & S1 & S2 & S3 & S4 & S5 & S6 & S7 & S8 & s9 & $\begin{array}{l}\text { Total por grupo } \\
\text { de ocupación }\end{array}$ \\
\hline G1 & 0,0 & 0,0 & 0,0 & 0,0 & 0,0 & 0,0 & 0,1 & 0,1 & 0,1 & 0,4 \\
\hline G2 & 0,1 & 0,0 & 0,1 & 0,1 & 0,3 & 0,3 & 0,1 & 0,7 & 5,3 & 7,0 \\
\hline G3 & 0,4 & 0,1 & 0,3 & 0,3 & 0,6 & 0,9 & 0,5 & 1,8 & 4,0 & 8,8 \\
\hline G4 & 0,4 & 0,2 & 0,5 & 0,4 & 0,9 & 1,7 & 2,5 & 2,5 & 1,9 & 10,9 \\
\hline G5 & 0,6 & 0,3 & 0,7 & 0,5 & 0,8 & 4,9 & 1,2 & 6,8 & 6,5 & 22,4 \\
\hline G6 & 28,1 & 0,3 & 0,3 & 2,8 & 1,0 & 1,2 & 0,3 & 0,4 & 1,4 & 35,9 \\
\hline G7 & 0,6 & 0,3 & 0,8 & 1,6 & 15,6 & 1,3 & 0,6 & 1,2 & 2,2 & 24,2 \\
\hline G8 & 1,8 & 0,3 & 0,5 & 0,8 & 1,3 & 1,2 & 3,9 & 0,8 & 0,7 & 11,4 \\
\hline G9 & 63,5 & 1,0 & 2,1 & 7,5 & 8,4 & 5,1 & 2,6 & 8,2 & 8,2 & 106,6 \\
\hline $\begin{array}{l}\text { Total por } \\
\text { sector }\end{array}$ & 95,6 & 2,6 & 5,4 & 14,1 & 29,0 & 16,7 & 11,7 & 22,4 & 30,2 & 227,7 \\
\hline
\end{tabular}

Fuente: Elaboración propia. (Leyenda: ver Tabla 1).

\section{APLICACIÓN DE LA METODOLOGÍA PROPUESTA. SIMULACIÓN DE IMPACTOS SECTORIALES ESPECÍFICOS DE LA CRISIS EN LA CONTRATACIÓN}

La metodología presentada en el apartado anterior y la obtención de la matriz $\mathbf{Z}$ (Tabla 4) permiten estimar, mediante simulación, los efectos de la actual crisis económica en sus primeros años (ya que los datos se basan en la estructura productiva de 2005). Puesto que, como se observa en la Tabla 2, los 
sectores con mayor retroceso en su actividad han sido los industriales y, muy especialmente, la Construcción, cabe preguntarse qué evolución habría sufrido la contratación en Andalucía si estos sectores no hubiesen entrado en recesión o, cuando menos, se hubiesen comportado como el conjunto de la economía (obviando en este caso que dicho comportamiento hubiese modificado dicho crecimiento promedio). Dado que el conjunto de la economía tiene una tasa de crecimiento cercana a cero en el período, simulamos a continuación un escenario en el que, tanto Industria, como Construcción tienen crecimiento nulo.

Para ello se ha aplicado la metodología propuesta, obteniendo las estimaciones de sus efectos en la contratación por ramas de actividad y por grupos de ocupación. Estos escenarios se han construido suponiendo, tanto para las Ramas industriales, como para Construcción, que su crecimiento en los años 2007, 2008 y 2009 fue, respecto a 2006, nulo (estancamiento). La Tabla 5 resume los resultados obtenidos.

\section{Tabla 5}

Simulación de los contratos realizados entre 2007 y 2009 en Andalucía, bajo supuesto de crecimiento nulo sectorial (Construcción e Industria).

Variación porcentual respecto a la situación real

\begin{tabular}{|l|cc|}
\cline { 2 - 3 } \multicolumn{1}{l|}{} & Construcción & Industria \\
\hline Total de contrataciones & 11,45 & 11,00 \\
\hline Dirección de las empresas y de las administraciones públicas & 16,59 & 16,38 \\
\hline Técnicos y profesionales científicos e intelectuales & 4,43 & 4,25 \\
\hline Técnicos y profesionales de apoyo & 11,53 & 11,37 \\
\hline Empleados de tipo administrativo & 20,77 & 20,54 \\
\hline $\begin{array}{l}\text { Trabajadores de los servicios de restauración, personales, protección } \\
\text { y vendedores de los comercios }\end{array}$ & 9,18 & 9,15 \\
\hline Trabajadores cualificados en la agricultura y en la pesca & 9,38 & 10,03 \\
\hline $\begin{array}{l}\text { Artesanos y trabajadores cualificados de las industrias } \\
\text { manufactureras, la construcción, y la minería, excepto los operadores } \\
\text { de instalaciones y maquinaria }\end{array}$ & 26,55 & 23,43 \\
\hline Operadores de instalaciones y maquinaria y montadores & 17,87 & 17,62 \\
\hline Trabajadores no cualificados & 5,97 & 5,94 \\
\hline Agricultura y pesca & 3,94 & 4,75 \\
\hline Industrias extractivas & 44,34 \\
\hline Producción y distribución de energía eléctrica, gas y agua & 15,96 & 46,73 \\
\hline Industria manufacturera & 14,24 & 17,03 \\
\hline Construcción & 29,83 & 16,26 \\
\hline Comercio & 25,38 \\
\hline Transporte y comunicaciones & 30,89 & 30,85 \\
\hline Otros servicios destinados a la venta & 14,27 & 14,01 \\
\hline Servicios no destinados a la venta & 9,70 & 9,64 \\
\hline
\end{tabular}

Fuente: Elaboración propia. 
En cuanto a la interpretación de los resultados presentados en esta tabla, hay que tener siempre presente que la mayor cantidad de contratos se da en Agricultura y pesca, y en Trabajadores no cualificados, que son los menos afectados en las simulaciones realizadas, con un impacto en las contrataciones inferior al $6 \%$, lo que explica una media tan baja, a pesar de que los resultados de casi todos los sectores y grupos de ocupación son muy superiores a la media para ambos análisis. Los resultados muestran como una evolución del sector de la Construcción igual a cero, es decir, si la el sector simplemente se hubiese estáncado en el periodo de análisis, hubiera permitido un 11,5\% más de contratación en la economía de la región (alrededor de 1.250 .000 contratos adicionales ${ }^{2}$ entre 2007 y 2009). Este incremento de los contratos se hubiese centrado, como era de esperar, en el propio sector $(31,8 \%)$ y en el grupo de ocupación Artesanos y trabajadores cualificados... (26,6\%), que recoge a los trabajadores especializados en el mismo. También destaca el crecimiento de los Operadores de instalaciones y maquinaria y montadores $(17,9 \%) \mathrm{y}$ de los Empleados de tipo administrativo (20,8\%). Por otra parte, hay que señalar, dentro de las ramas de actividad, el aumento en Industrias Extractivas, cercano al $45 \%$ y superior al del propio sector, y en Comercio (30,9\%), lo que nos indica el importante efecto arrastre que la Construcción tiene en Andalucía para ambos sectores.

Como ya hemos señalado, destaca el escaso impacto que en estos escenarios se hubiera producido sobre el grupo de ocupación de Trabajadores no cualificados (alrededor del 6\% de incremento sobre la situación real), seguramente debido a que la evolución real en los mismos ha sido mejor que la media debido a su absorción en los mercados de trabajo del sector primario. Precisamente los Trabajadores cualificados en agricultura y pesca incrementarían sus contrataciones cerca del 9,4\%, algo similar a lo que se obtiene para los Trabajadores de los servicios de restauración,... (9,2\%). Este último dato, contrasta con el importante incremento de contratos que se obtendría en la rama de Comercio.

La realización del mismo tipo de simulación, pero ahora con las Ramas Industriales, da lugar a unos resultados similares para el total de contratos, aunque con algunas diferencias en lo que a ramas productivas se refiere. Esto es debido a que la simulación en este caso afecta conjuntamente a los sectores de Industrias extractivas, Producción y distribución de energía eléctrica, gas y agua e Industrias manufactureras, en los que los efectos sobre la contratación son más elevados que en la simulación anterior. Así, en el caso de que consideremos un estancamiento en el crecimiento de estos sectores, el total de contratos hubiera sido, para el conjunto del periodo 2007 y 2009, un $11 \%$ mayor, cifra ligeramente inferior a la obtenida cuando analizamos el impacto que tiene un

\footnotetext{
${ }^{2}$ Cuando hablamos de contratos hay que tener presente que no siempre son contratos duraderos, sino que pueden ser temporales de corta duración, tan frecuentes en algunos sectores como la Agricultura. Es por ello que hay que tomar esta cifra con cierta precaución.
} 
estancamiento en el sector de la Construcción. En este caso hay que resaltar el gran impacto sobre las contrataciones realizadas por la rama de Comercio (casi un 31\%), así como el impacto sobre el sector de la Construcción. El impacto sobre el propio sector industrial es también importante, sobre todo el efecto sobre el número de contratos realizados por la Industria extractiva. Por otra parte, destaca de nuevo el bajo impacto sobre Trabajadores no cualificados y sobre Agricultura y pesca, sobre todo teniendo en cuenta la importancia que la industria alimentaria tiene en la región y por la que cabría esperar un mayor impacto sobre el sector primario.

Para finalizar este análisis haremos una reflexión general contextualizada en el entorno nacional. Entre 2007 y 2011 la destrucción de empleo en España ha sido de 2,7 millones de trabajadores expulsados del mercado laboral, de los cuales 1,4 y 0,7 millones han sido en los sectores analizados en este epígrafe (Construcción e Industria), es decir, prácticamente el 77\% del empleo destruido. Andalucía ha sido una de las regiones que más ha contribuido a este dudoso honor. De esta forma, a nivel regional, en estos cinco años se ha producido un cambio sustancial en el porcentaje de empleo sectorial: los dos sectores estudiados han descendido en un $8,11 \%$ y $1,21 \%$, respectivamente, en cuanto a su distribución sobre el empleo total. Por lo tanto los resultados aquí expuestos, aunque claramente negativos, son conservadores, al hacerse la simulación con un escenario de crecimiento nulo, ni siquiera de recesión o crecimiento negativo. En cualquier caso, la desagregación por tipología de contratación arroja luz suficiente de la situación que atraviesa el mercado laboral andaluz.

\section{CONCLUSIONES}

En un momento como el actual, en el que el mercado de trabajo está en el punto de mira, tanto por las dificultades que está teniendo la economía española para crear empleo en comparación con nuestros socios europeos, como por la falta de acuerdo entre los agentes sobre cuáles son las mejores medidas para reducir el desempleo, se hace necesaria la búsqueda de herramientas que contribuyan a evaluar el impacto que las diferentes medidas tendrán en la economía española.

El análisis seguido en este trabajo pretende, por una parte, presentar una metodología que pueda servir de herramienta en la toma de decisiones de política económica encaminadas a la creación de empleo en determinados colectivos de ocupaciones o categorías profesionales. La obtención de la matriz $\mathbf{Z}$ de impactos en la contratación causados por shocks exógenos (inversión o demanda final pública o privada, exportaciones, etc.) permite aproximar qué tipos de ocupaciones se pueden ver beneficiadas de actuaciones específicas en determinados sectores. Ya se ha señalado, en el resumen de los principales resultados, que los efectos de impacto de shocks exógenos en distintas ramas de actividad, no sólo 
tienen su reflejo en aquellas categorías profesionales que puedan caracterizar más su ocupación, sino que, mediante la transmisión de efectos a través del flujo circular de la renta, inciden en las contrataciones de otros colectivos. Esto permite combinar acciones y políticas multisectoriales en la búsqueda del objetivo final de la creación de empleo.

Por otra parte, se ha ilustrado el uso de este tipo de análisis con su aplicación a la economía andaluza, mostrando la importancia de la caída de algunos sectores en el conjunto de las contrataciones y en algunos grupos de ocupación específicos, mostrando que el shock del sector de la Construcción, por sí solo, es responsable del descenso de algo más de un $11 \%$ en el número de contratos realizados en Andalucía entre los años 2007 y 2009. Igualmente se ha evaluado el impacto de un shock sobre las ramas industriales, que arroja unos resultados similares con un aumento de la contratación en un $11 \%$. En cuanto a los resultados detallados, además del impacto esperado sobre los propios sectores sobre los que se realiza el shock, destaca el fuerte impacto sobre la rama de Comercio, tan importante para la región.

En cuanto a los grupos de ocupación, el impacto es especialmente intenso en dos grupos de ocupación, Artesanos y trabajadores cualificados.... y Empleados de tipo administrativo. Finalmente, destaca el escaso impacto de los escenarios valorados sobre el grupo de ocupación de Trabajadores no cualificados, seguramente debido a que la evolución real en los mismos ha sido mejor que la media debido a su absorción en los mercados de trabajo del sector primario.

Los resultados obtenidos muestran el importante efecto arrastre que tiene el sector de la Construcción en la economía andaluza, incluso superior al de las ramas industriales, aun tratándose éste último de un sector con un peso considerablemente mayor en la economía de la región.

En resumen, el uso de modelos multisectoriales como el aquí presentado puede ser un instrumento útil para analizar los efectos de la actividad económica sobre el mercado de trabajo y plantear escenarios de trabajo que lleven a la obtención de soluciones eficientes y eficaces. En este sentido, la metodología de análisis presentada puede aplicarse en el resto de regiones españolas (siempre y cuando dispongan de alguna MCS o, cuando menos, de un marco input-output) o a nivel nacional, permitiendo incluso su extensión a comparaciones internacionales.

\section{REFERENCIAS BIBLIOGRÁFICAS}

ARIZA, A.; TRONCOSO, D.; GARCÍA PÉREZ, J.I. (2011). Análisis del Mercado de trabajo de España y Andalucía: Encuesta de Población Activa. Cátedra BBVA de Análisis Económico. 
CÁMARA, Á.; FLORES, M.; FUENTES, P. D. (2011). "Análisis económico y medioambiental del sector eléctrico en España". Estudios de Economía Aplicada, vol. 29-2, págs. 493-514.

CARDENETE, M. A.; FUENTES, P. D.; POLO, C. (2008). "Análisis de intensidades energéticas y emisiones de $\mathrm{CO}_{2}$ a partir de la Matriz de Contabilidad Social de Andalucía para el año 2000" Economía Agraria y Recursos Naturales, vol. 8(2), págs. 31-48.

CARDENETE, M. A.; FUENTES, P. D.; LÓPEZ, J. (2010). Análisis del mercado laboral en Andalucía. Sectores claves estratégicos. CC.OO. Andalucía.

CARDENETE, M. A.; FUENTES, P. D. (2009). "Cambios en la estructura económica andaluza, 1990-2005: Un análisis a partir de las Matrices de Contabilidad Social regionales", XXXV Reunión de estudios regionales, Valencia, 26 y 27 de noviembre de 2009.

INSTITUTO DE ESTADÍSTICA Y CARTOGRAFÍA DE ANDALUCÍA. Contabilidad Regional Anual de Andalucía. www.juntadeandalucia.es/ institutodeestadisticaycartografia

KEHOE. T.J. MANRESA. A. POLO. C. SANCHO. F. (1988). "Una Matriz de Contabilidad Social de la Economía española". Estadística Española, vol. 30. № 117, pp. 5-33.

OBSERVATORIO ARGOS (varios años). Servicio Andaluz de Empleo. Consejería de Empleo. Junta de Andalucía.

POLO, C.; ROLAND-HOLST, D. Y SANCHO, F. (1991). "Descomposición de multiplicadores en un modelo multisectorial: una aplicación al caso español". Investigaciones Económicas, v. XV n¹, pp. 53-69.

POLO, C.; VALLE, E. (2011). "The Weight of Tourism in the Balearic Islands: 1983-1997-2004". Estudios de Economía Aplicada, vol. 29-3, págs. 737-754.

PYATT, G. Y ROUND, J. (1979). "Accounting and Fixed Price Multipliers in a Social Accounting Framework", Economic Journal, n² 89, pp. 850-873.

SISTEMA DE INFORMACIÓN DE LOS SERVICIOS PÚBLICOS DE EMPLEO (SISPE). SERVICIO PÚBLICO DE EMPLEO ESTATAL. MINISTERIO DE EMPLEO Y SEGURIDAD SOCIAL. Bases estadísticas (varios años). www.sepe.es.

STONE. R. (1962). A Social Accounting Matrix for 1960. A Programme for Growth. Edit. Chapman and Hall Lid, London. 
